JGG 2021;69:177-187

doi: $10.36150 / 2499-6564-N 374$

\title{
Cardiac amyloidosis awareness among residents/specialists in Geriatrics: an Italian national survey
}

Diana Lelli ${ }^{1}$, Claudio Pedone ${ }^{1}$, Dario Leosco², Raffaele Antonelli Incalzi', for the Società Italiana di Gerontologia e Geriatria

${ }^{1}$ Unit of Geriatrics, Campus Bio-Medico University of Rome, Rome, Italy; ${ }^{2}$ Department of Translationl Medical Sciences, University of Naples "Federico II", Naples, Italy

Objective. Cardiac amyloidosis (CA) among older adults is less infrequent than usually considered, and often underdiagnosed. Geriatricians' awareness and knowledge of CA is unknown. Objectives of the study are to assess actual CA knowledge among residents/specialists in Geriatrics, and their usual clinical practice in managing suspected CA.

Methods. In this Nation-wide survey carried out by the Italian Society of Gerontology and Geriatrics in April-August 2020, we administered to residents/specialists in Geriatrics a questionnaire divided in three sections: socio-demographical, procedural and knowledge. Results were stratified by qualification (resident/specialist) and by performance (best/worst performers).

Results. 289 residents and 104 specialists participated. The overall proportion of correct answers was 57.9\% (58.4\% residents, $56.1 \%$ specialists); knowledge about clinical (89\% of correct answers, 91\% residents and $84 \%$ specialists, $P=0.062)$, ECG (72\% of correct an-

Received: March 25, 2021

Accepted: April 20, 2021

\section{Correspondence}

Diana Lelli

Unit of Geriatrics, Campus Bio-Medico University of Rome, via Alvaro del Portillo 21, 00128 Rome, Italy. Tel. +3906 225411495. Fax +3906

22541456. E-mail: d.lelli@unicampus.it

How to cite this article: Lelli D, Pedone C, Leosco D, et al. Cardiac amyloidosis awareness among residents/ specialists in geriatrics: an Italian national survey. Journal of Gerontology and Geriatrics 2021;69:177-187. https://doi. org/10.36150/2499-6564-N374

(C) Copyright by Società Italiana

di Gerontologia e Geriatria (SIGG)

\section{(c) (i) $(9)$}

\section{OPEN ACCESS}

This is an open access article distributed in accordance with the CC-BY-NC-ND (Creative Commons Attribution-NonCommercial-NoDerivatives 4.0 International) license. The article can be used by giving appropriate credit and mentioning the license, but only for non-commercial purposes and only in the original version. For further information: https://creativecommons.org/licenses/by-nc-nd/4.0/deed.en swers, no differences between groups), and echocardiographic (86\% of correct answers, no differences between groups) signs of CA was good. However, only $8 \%$ participants knew how to diagnose ATTRwt CA (no differences between groups), and $19 \%$ which diuretic is indicated in CA (22\% residents, $13 \%$ specialists, $\mathrm{P}=0.069)$. Only $25 \%$ of the participants knew the natural history of ATTRwt (19\% residents, $41 \%$ specialists, $P<0.001$ ), and $37 \%$ was aware of the target of the ATTR treatment. Prevalence of CA was underestimated by $57 \%$ specialists and $37 \%$ residents $(P=0.001)$.

Conclusions._Knowledge of CA among residents/specialists in Geriatrics is uncomplete. Education campaigns on this topic are desirable, to improve physicians' awareness of CA, thus reducing the number of potential misdiagnosis/delay in correct diagnoses.

Key words: survey, older adults, cardiac amyloidosis, amyloidosis, geriatrician

\section{INTRODUCTION}

Amyloidosis is a systemic chronic and progressive disease characterized by extracellular deposition of amyloid, a fibrillar material derived from various precursor proteins, that leads to organ failure ${ }^{1}$. Although there 
are more than 30 different precursor proteins implicated in various amyloid diseases, only 2 types account for over $95 \%$ of all cardiac amyloidosis (CA), characterized by diffuse amyloid fibril deposition in the heart causing thickening of both ventricles and appearing phenotypically as hypertrophic cardiomyopathy: immunoglobulin light chain amyloidosis (AL) and transthyretin amyloidosis (ATTR) ${ }^{2}$. AL is a rare disease characterized by a clonal plasma cell disorder due to the overproduction and misfolding of antibody light chain fragments, while ATTR is due to misfolding of the liver-derived precursor protein transthyretin (TTR), either as a hereditary mutant (ATTRm) or as an acquired wild-type variant (ATTRwt) ${ }^{3}$. The latter is the most common and typically has its clinical onset in advanced age, with clinical signs of heart failure ${ }^{4}$.

Although CA has been considered a rare disease for a long time, recent evidence show that its prevalence has been largely underestimated. In the US, both incidence and prevalence increased over the years, up to a prevalence of about 55 cases per 100,000 persons/ year in $2012^{5}$. In older people, the prevalence is probably higher, as reported by several autopsy studies indicating the presence of ATTRwt myocardial deposits in $20-25 \%$ of octogenarians ${ }^{6}$, and up to $37 \%$ in patients older than $>95$ years ${ }^{5}$.

Being a relatively rarely diagnosed syndrome, the underdiagnosis may be related to the poor awareness of this disease among physicians, bringing to late or no diagnosis. However, little is known about the real knowledge of CA among physicians: a survey on physicians' attitudes in amyloidosis treatment has been recently performed $^{7}$, while only one study assessed knowledge among cardiologists ${ }^{8}$.

Given the late clinical manifestations of ATTRwt CA and its common presentation as heart failure, a clinical condition frequently treated by geriatricians ${ }^{9}$, it is pivotal for these specialists to suspect this disease when indicated. Therefore, assessing the knowledge about CA among specialists and trainees in Geriatrics, would allow to identify the educational needs in all the phases of these physicians' training.

The aim of this nationwide Italian survey was to assess the actual knowledge about CA among residents and specialists in Geriatrics, in order to plan instructional programs on this topic, if needed. Secondary aim was to evaluate usual clinical practice in managing suspected CA by these physicians.

\section{METHODS}

This National survey was ideated and carried out in Italy from April to August 2020 by the Italian Society of
Gerontology and Geriatrics (Società Italiana di Gerontologia e Geriatria, SIGG).

The study questionnaire was developed by a consensus group including three Geriatricians expert in CA. It included three sections, using clinical vignettes and multiple-choice answers: the first section collected demographic data, information on working geographical data and setting; the second section included procedural information, exploring education on amyloidosis and clinical attitudes in the management of patients with suspected or diagnosed CA; the third section explored the knowledge about the epidemiology, clinical features, diagnosis, and management of CA according to the most recent evidences available on this topic. In the design of the latest section, a particular interest was given to the most common types of CA, especially to the ATTRwt, the most widespread in older adults (Supplemental text 1).

This kind of questionnaire proved useful to interpret current practice on the basis of individual experience and culture in previous surveys on geriatric competences among Nephrologists ${ }^{10}$, nephrological competences among Geriatricians ${ }^{11}$, vaccines and vaccination in older people ${ }^{12}$, and heart failure ${ }^{9}$.

The secretary of the Society sent an email with an electronic copy of the survey to all the Italian Directors of Internship Programs in Geriatrics who were registered in the membership list of the Society, and to all the members of the National and Regional Governing Councils of the Society. They were invited to participate to the survey and to share the e-mail with the attached survey to all their colleagues being specialists or fellows in Geriatrics.

\section{StATISTICAL ANALYSIS}

Quantitative variables were reported using mean and standard deviation, while categorical ones were reported using proportions. All the analyses were performed on the whole sample and stratified according to qualification (specialist vs. resident). Comparison between groups were performed using the t-test for continuous variables and the chi-squared test for proportions.

In the third section, we calculated the proportion of correct answers for each question (only one possible correct answer), and a pooled proportion of correct answers.

In order to identify the characteristics of participants needing more educational support, we performed a comparison between participant in the highest (best performers) and lowest (worst performers) quartile of percentage of correct answers.

All the analyses were performed using R software, version 3.6.1 (Vienna, Austria). 


\section{RESULTS}

\section{General Characteristics of the population}

A total of 393 physicians, 289 residents and 104 specialists, participated to the survey. Mean age of the study population was 34.5 years (SD 9.2); 35\% were male. With respect to specialists, residents were younger (45.3 years, SD 11.1 vs 30.6 years, SD 3.6) and less frequently male (42.3 vs $32.3 \%, P=0.008$ ), more frequently working in acute care ward (58.7 vs $90.3 \%)$, and less frequently in nursing home (13.5 vs $0 \%)$, or in outpatient clinic (14.4 vs 5.2\%) $(P<0.001)$ (Tab. I). The distribution of specialists participating to the survey across National macro-areas is reported in Figure 1. Forty-five percent of participants worked in Southern Italy, while $32.8 \%$ in Central and $22.1 \%$ in Northern Italy, with a higher prevalence of residents in Northern (25.3 vs 13.5\%) Italy, and a slight lower prevalence in Central and Southern Italy $(P=0.046)(T a b . ~ I)$.

\section{EducATION AND CLINICAL ATTITUdES FOR CA OF THE POPULATION}

Most of the participants obtained information on CA from Pubmed (78.1\%, with no differences between residents and specialists), or by individual web search (54.2\%; residents 58.8 vs specialists $41.3 \%, \mathrm{P}=0.003$ ); specialists more frequently studied using material provided by pharmaceutical company representatives (21.2 vs $5.2 \%, P<0.001)$, and using International scientific journals (44.2 vs 30.8\%, $P=0.019$ ). Globally, 38.7\% of the participants had previously managed patients with CA $(60.6 \%$ among specialists and $30.1 \%$ among residents), $20.6 \%$ of which were patients visited for

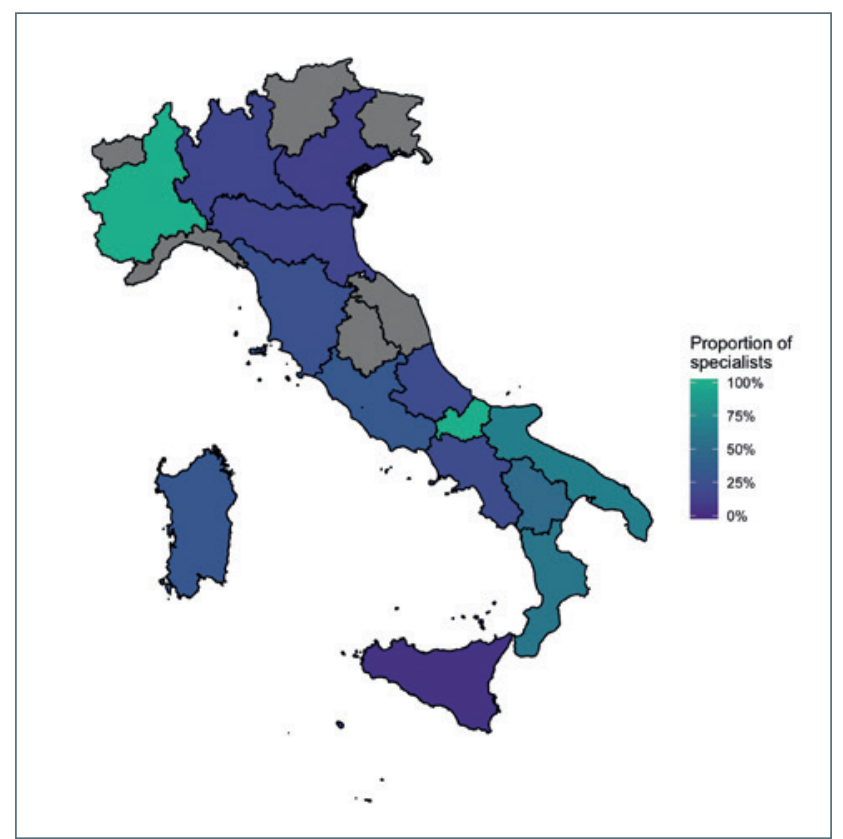

Figure 1. Participation to the survey. Distribution of specialists participating to the survey across National macro-areas. Grey areas indicate regions without participants to the survey.

symptoms of heart failure, and $20.1 \%$ visited for other diseases, and in $13 \%$ of cases it was a first diagnosis of CA. About $68 \%$ of participants $(74.8 \%$ of the specialists and $65 \%$ of the residents, $P=0.191$ ) prescribed second level exams with suspected CA regardless of age and comorbidities.

In case of CA with cardiologic symptoms, 59\% preferred to manage personally older patients with multimorbidity (no differences between groups), and $41.2 \%$ asked

Table I. General characteristics of the population.

\begin{tabular}{|c|c|c|c|c|}
\hline & $\begin{array}{c}\text { Residents } \\
\text { N: } \mathbf{2 8 9}\end{array}$ & $\begin{array}{c}\text { Specialists } \\
\text { N: } 104\end{array}$ & $\begin{array}{c}\text { All } \\
\text { N: } 393\end{array}$ & $\mathbf{P}$ \\
\hline Age, years, mean (SD) & $30.6(3.6)$ & $45.3(11.1)$ & $34.5(9.2)$ & $<0.001$ \\
\hline Male sex N (\%) & $93(32.3)$ & $44(42.3)$ & $137(34.9)$ & 0.008 \\
\hline \multicolumn{5}{|l|}{ Type of practice, N (\%) } \\
\hline Outpatient clinic & $15(5.2)$ & $15(14.4)$ & $30(7.6)$ & $<0.001$ \\
\hline Acute care ward & $261(90.3)$ & $61(58.7)$ & $322(81.9)$ & \\
\hline Rehabilitation & $3(1)$ & $2(1.9)$ & $5(1.3)$ & \\
\hline Nursing home & $0(0)$ & $14(13.5)$ & $14(3.6)$ & \\
\hline Other & $7(2.5)$ & $9(8.6)$ & $16(3.1)$ & \\
\hline No answer & $3(1)$ & $3(2.9)$ & $6(1.5)$ & \\
\hline \multicolumn{5}{|l|}{ Geographical area, N (\%) } \\
\hline North & $73(25.3)$ & $14(13.5)$ & $87(22.1)$ & 0.046 \\
\hline Center & $91(31.5)$ & $38(36.5)$ & $129(32.8)$ & \\
\hline South & $125(43.3)$ & $52(50)$ & $177(45)$ & \\
\hline
\end{tabular}


for a cardiologic visit to all patients, independently from age or other characteristics (residents 40.1 vs specialists 44.2, $\mathrm{P}=0.543$ ).

When visiting patients with features suggestive for CA, screening exams for amyloidosis were prescribed by only $55.1 \%$ of the participants, while $26.5 \%$ of physicians preferred to firstly treat heart failure symptoms, and prescribed a screening for CA only in case of treatment failure (no differences between groups).

Only $50.9 \%$ of participants reported that amyloidosis was included in teaching activities during medical school, and $12 \%$ reported that it was never included in their training (8.3 among residents and $22.3 \%$ among specialists) $(P<0.001)$ (Supplemental Table I).

\section{KNOWLEDGe ABOUt CA OF THE STUDY SAMPLE}

Overall, the rate of correct answers was 57.9\% (58.4\% among residents and $56.1 \%$ among specialists). However, this result stems from a sharp difference between general knowledge of CA, fair, and that of the diagnostic pathway, such as diagnostic criteria of amyloidosis or scintigraphy interpretation, which was poor. Indeed, the knowledge about clinical (89\% of correct answers, 91\% among residents and $84 \%$ among specialists, $\mathrm{P}=0.062)$, ECG (72\% of correct answers, with no differences between groups), and echocardiographic (86\% of correct answers, 84\% among residents and 91\% among specialists, $P=0.091$ ) signs of $C A$ was good. Comparable figures were obtained for the knowledge of the clinical history leading to suspect CA $(90 \%$ of correct answers, 96\% among residents and 75\% among specialists), and the diagnostic role of immunofixation in suspected CA (87\% of correct answers, no differences between groups). On the other hand, very poor was the knowledge about how to diagnose ATTRwt CA (8\% of correct answers, no differences between groups), or about diuretic treatment in patients with CA $119 \%$ of correct answers, $22 \%$ among residents and 13\% among specialists, $P=0.069$ ). Furthermore, only $25 \%$ of the participants knew the natural history of ATTRwt (19\% among residents and $41 \%$ among specialists, $\mathrm{P}<0.001$ ), and only $38 \%$ how to perform differential diagnosis of CA using bone scintigraphy (30\% among specialists and $41 \%$ among residents, $\mathrm{P}=0.065)$, and only $37 \%$ of participants was aware of the target of the ATTR treatment. Finally, the prevalence of CA was underestimated by $57 \%$ specialists and $37 \%$ residents (P = 0.001) (Tab. II).

Comparing "best" with "worst" performers, there were no differences in age, sex, and status, despite the former group was slightly younger and included a majority of residents. Best performers worked more frequently in

Table II. Summary of correct answers per issue.

\begin{tabular}{|c|c|c|c|c|}
\hline Questions & Residents (\%) & Specialists (\%) & All (\%) & $\mathbf{P}$ \\
\hline Clinical signs of CA among older adults & 91 & 84 & 89 & 0.062 \\
\hline ECG typical signs of CA & 72 & 69 & 72 & 0.616 \\
\hline Echocardiographic typical signs of CA & 84 & 91 & 86 & 0.091 \\
\hline Prevalence of CA & 37 & 57 & 43 & 0.001 \\
\hline Common arrhythmic/conduction system defects in CA & 69 & 60 & 67 & 0.146 \\
\hline Common features of amyloidosis & 70 & 65 & 69 & 0.487 \\
\hline Amyloidosis AL & 61 & 46 & 57 & 0.011 \\
\hline Amyloidosis ATTR & 65 & 62 & 64 & 0.656 \\
\hline Amyloidosis ATTRwt & 19 & 41 & 25 & $<0.001$ \\
\hline Role of serum and urinary immunofixation in suspected CA & 86 & 89 & 87 & 0.423 \\
\hline Instrumental diagnosis of amyloidosis & 58 & 54 & 57 & 0.570 \\
\hline $\begin{array}{l}\text { Management of older patient with atrial fibrillation and new } \\
\text { onset of heart failure with history of carpal tunnel }\end{array}$ & 96 & 75 & 90 & $<0.001$ \\
\hline Diagnosis of amyloidosis ATTRwt & 9 & 6 & 8 & 0.403 \\
\hline Diuretic treatment in $\mathrm{CA}$ & 22 & 13 & 19 & 0.069 \\
\hline Drugs not recommended in CA & 67 & 75 & 69 & 0.210 \\
\hline The target of amyloidosis ATTR treatment & 40 & 30 & 37 & 0.100 \\
\hline Clinical classification of amyloidosis ATTR & 41 & 53 & 45 & 0.050 \\
\hline Prevalence of association between aortic valve stenosis and CA & 56 & 42 & 52 & 0.024 \\
\hline $\begin{array}{l}\text { Differential diagnosis of CA from myocardial uptake in bone } \\
\text { scintigraphy }\end{array}$ & 41 & 30 & 38 & 0.065 \\
\hline Tafamidis mechanism of action & 85 & 80 & 84 & 0.330 \\
\hline
\end{tabular}

Abbreviations: AL: light chain; ATTRwt: transthyretin wild type; CA: cardiac amyloidosis; ECG: electrocardiogram 
Southern Italy (59 vs 33\%, $\mathrm{P}<0.001$ ) and in acute care wards (90 vs $81 \%, P=0.028$ ), and used more frequently Pubmed to study CA (84.5 vs $73.3 \%, P=0.068$ ). There were no differences in clinical experience of CA, while best performers more frequently prescribed second level exams in the case of suspected CA, independently from age and comorbidities (75.3 vs $60.7 \%$, $P=0.048$ ). Finally, best performers more frequently had studied amyloidosis both during medical school and the internship program (30.5 vs 16.7\%, $P=0.045)$.

\section{DISCUSSION}

Overall, the participants in this Italian National survey had an uncomplete knowledge of CA, independently from their status of specialist or resident in Geriatrics: despite the good theoretical education on clinical features of this disease, they lacked information on how to diagnose it, especially the ATTRwt, that is the most common form of CA in older adults.

Our data are in line with the results reported by Mircsof et al among 72 Cardiologists working in Switzerland, that showed a wide variation in knowledge and in diagnostic tests used during differential diagnosis of ATTR $\mathrm{CA}$, and concluded that Cardiologists in that country would benefit from information on the latest advances in ATTR ${ }^{8}$.

A key result of our survey is the evidence that most physician, independently from their status of resident or specialist, underestimate the prevalence of CA. This indicates that improving the awareness of the physician about the frequency of this disease is pivotal: other studies have shown that there is a delay of up to 39 months from first presentation of cardiac symptoms to CA diagnosis and over 4 years if considering ATTRwt ${ }^{13}$. Furthermore, there is inadequate knowledge on how to diagnose CA and to interpret bone scintigraphy results. This non-invasive diagnostic technique, easy to perform also among older adults ${ }^{14}$, has $99 \%$ sensitivity and $86 \%$ specificity for cardiac ATTR ${ }^{15}$ and has been included as a screening tool for ATTR-CA in the 2016 European Society of Cardiology guidelines for the treatment of heart failure ${ }^{16}$.

Worthy of note also are the really poor physicians' awareness of how to diagnose ATTRwt, the most common form of CA among older adults ${ }^{4}$, and the relatively low number of patients sent for specific treatments (already documented among Cardiologists ${ }^{8}$ ), probably due to the poor knowledge of which is the target for ATTRwt treatment.

All these issues are pivotal considering that new and effective treatments for CA have become available in the latest years. For example, Tafamidis, a tetramer stabilizer that binds with high affinity and selectivity to the thyroxine site of TTR slowing dissociation of TTR tetramers into monomers and preventing aggregation in amyloid fibrils, has been shown to be effective in reducing cardiovascular-related hospitalizations and allcause mortality; it also improves physical performance and quality of life of patients affected by TTR $C A{ }^{17}$, and has been approved for treatment of both wild-type and mutated TTR CA ${ }^{18}$. Other molecules, such as AG10, another transthyretin tetramer stabilizer, and Patisiran, a transthyretin synthesis inhibitor, have shown promising results, and, currently, are under investigation in phase III trials ${ }^{18}$. Furthermore, it should be considered that awareness on CA could help, if diagnosed or even only suspected, in revising carefully the cardiologic drugs used to treat symptoms of heart failure. Indeed, selected drugs, such as non-dihydropyridine calcium channel-blockers and digoxin, could have important adverse effects when used in patients affected by $\mathrm{CA}^{3}$. Interestingly, our survey documented that participants having the best performance were slightly younger and more frequently resident, and that there were no differences with respect to worst performers in their personal experience of $\mathrm{CA}$, and best performers more frequently studied CA both during their MD and fellowship. These physicians were also those that more frequently prescribed second level exams for suspected amyloidosis. Thus, our findings once more highlight the importance of an adequate education in CA to improve physicians' awareness of this disease and, thus, to suspect CA and prescribe second levels exams.

Our study has some weaknesses: firstly, the sampling of the study population mostly included physicians working in University settings, thus leading to a plausible overestimation of the guidelines' knowledge from the whole cohort of the Italian Geriatricians ${ }^{19}$. Nevertheless, this was an obvious selection condition to focus on residents in Geriatrics. Second, it was a questionnaire developed ad hoc for the purpose of this survey, and its clinimetric properties were not evaluated. However, previous experience is consistent with this kind of questionnaire having classificatory properties able to gain insight into the dynamic underlying physician's awareness of and practice with selected different conditions $^{9,12}$. Furthermore, the main aim of the survey was to have an overview of the approach to CA and not a punctual assessment of this disease.

\section{CONCLUSIONS}

This Italian National survey highlighted that the knowledge of CA among both residents and specialists in Geriatrics is uncomplete, and, thus, education campaigns on this 
Table III. Main characteristics of the participants according to their performance.

\begin{tabular}{|c|c|c|c|}
\hline & Worst performers & Best performers & $\mathbf{P}$ \\
\hline Age, years, mean (SD) & $35.6(10.2)$ & $33.4(7.3)$ & 0.071 \\
\hline Male sex, \% & 41 & 33 & 0.295 \\
\hline Resident, \% & 70 & 81 & 0.075 \\
\hline Region of working, \% & & & $<0.001$ \\
\hline Center & 24 & 27 & \\
\hline North & 42 & 14 & \\
\hline South & 33 & 59 & \\
\hline Type of practice, $\%$ & & & 0.028 \\
\hline Outpatient clinic & 9 & 2 & \\
\hline Acute care ward & 81 & 90 & \\
\hline Rehabilitation & 2 & 1 & \\
\hline Nursing home & 2 & 0 & \\
\hline No answer & 0 & 4 & \\
\hline \multicolumn{4}{|c|}{ Where do you study clinical features, diagnosis and treatment of cardiac amyloidosis (more than one answer possible)? } \\
\hline PubMed & 73.3 & 84.5 & 0.068 \\
\hline Web pages and publications of the Ministry of Health & 10.8 & 19.6 & 0.106 \\
\hline Congresses and training courses & 25 & 16 & 0.120 \\
\hline International Scientific journals & 28 & 33 & 0.466 \\
\hline Individual web search & 53 & 61 & 0.275 \\
\hline \multicolumn{4}{|l|}{ If you suspect cardiac amyloidosis: } \\
\hline $\begin{array}{l}\text { You always prescribe second level exams, independently of age and } \\
\text { comorbidities, with the exception of terminal patients }\end{array}$ & 60.7 & 75.3 & 0.048 \\
\hline You prescribe second level exams only for younger patients & 1.7 & 0 & \\
\hline $\begin{array}{l}\text { You prescribe second level exams only for patients with good } \\
\text { performance status and few comorbidities }\end{array}$ & 37.6 & 24.7 & \\
\hline \multicolumn{4}{|l|}{ In your training, amyloidosis was studied: } \\
\hline During the MD & 55.8 & 43.2 & 0.045 \\
\hline During the fellowship & 12.5 & 7.4 & \\
\hline Both during the MD and the fellowship & 16.7 & 30.5 & \\
\hline Never, only occasional information during congresses & 15 & 18.9 & \\
\hline \multicolumn{4}{|l|}{ In your clinical practice, heart failure is: } \\
\hline Very common (> $30 \%$ of cases) & 71.7 & 66 & 0.781 \\
\hline Common (10-30\% of cases) & 19.2 & 22.7 & \\
\hline Uncommon $(<10 \%$ of cases) & 7.5 & 8.2 & \\
\hline Very uncommon & 16.7 & 3.1 & \\
\hline
\end{tabular}

No significant differences were reported in experience with patients with cardiac amyloidosis, nor for their clinical management.

topic are desirable, with the aim of improving physicians' awareness of CA, thus reducing the number of potential misdiagnosis or delay in correct diagnoses, and improving quality of life and prognosis of these patients.

\section{Ethical consideration}

This research complies with the ethical rules for human experimentation stated in the Declaration of Helsinki.

\section{Acknowledgement}

We would like to acknowledge all the Directors of the Italian Internship programs in Geriatrics for their participation to the Survey.
We would like to acknowledge also Gilda Borselli and Letizia Scarpelli for their support for data management. The corresponding author has listed everyone who contributed significantly to the work and has obtained written consent from all contributors who are not authors and are named in the Acknowledgment section.

\section{Conflict of interest}

The authors have no conflicts of interest to disclose.

Author contributions

Diana Lelli, Claudio Pedone, Dario Leosco, and Raffaele Antonelli Incalzi contributed to the study conception 
and design and to the acquisition of data. Data analysis were performed by Diana Lelli, and were interpreted by all the authors. The first draft of the manuscript was written by Diana Lelli, and all authors commented on previous versions of the manuscript. All authors read and approved the final manuscript.

\section{Funding sources and sponsors' role}

This study was supported by a liberal, non-conditioning, educational grant by Pfizer Italia.

\section{References}

1 Wechalekar AD, Gillmore JD, Hawkins PN. Systemic amyloidosis. Lancet 2016;387:2641-2654. https://doi. org/10.1016/S0140-6736(15)01274-X

2 Maleszewski JJ. Cardiac amyloidosis: pathology, nomenclature, and typing. Cardiovasc Pathol 2015;24:343-350. https://doi.org/10.1016/j.carpath.2015.07.008

3 Donnelly JP, Hanna M. Cardiac amyloidosis: An update on diagnosis and treatment. Cleve Clin J Med 2017;84(12 Suppl 3):12-26. https://doi.org/10.3949/ccjm.84.s3.02

4 Ruberg FL, Grogan M, Hanna M, et al. Transthyretin amyloid cardiomyopathy: JACC state-of-the-art review. J Am Coll Cardiol 2019;73:2872-2891. https://doi.org/10.1016/j. jacc.2019.04.003

5 Gilstrap LG, Dominici F, Wang Y, et al. Epidemiology of cardiac amyloidosis - associated heart failure hospitalizations among fee-for-service medicare beneficiaries in the United States. Circ Heart Failure 2019;12:e005407. https://doi. org/10.1161/CIRCHEARTFAILURE.118.005407

6 Tanskanen M, Peuralinna T, Polvikoski T, et al. Senile systemic amyloidosis affects $25 \%$ of the very aged and associates with genetic variation in alpha2-macroglobulin and tau: a population-based autopsy study. Ann Med 2008;40:232239. https://doi.org/10.1080/07853890701842988

7 Milani P, Gertz MA, Merlini G, et al. Attitudes about when and how to treat patients with $\mathrm{AL}$ amyloidosis: an international survey. Amyloid 2017;24:213-216. https://doi.org/1 $0.1080 / 13506129.2017 .1370421$

8 Mircsof D. Diagnosis of amyloidosis: a survey of current awareness and clinical challenges among cardiologists in Switzerland. Cardiol Ther 2020;9:127-138. https://doi. org/10.1007/s40119-019-00160-8
9 Lelli D, Pedone C, Leosco D, et al. Management of heart failure: an Italian national survey on fellows/specialists in geriatrics. Aging Clin Exp Res 2020;32:2049-2055. https://doi.org/10.1007/s40520-020-01577-1

10 Aucella F, Brunori G, Dalmartello M, et al. Assessment of the geriatric competence and perceived needs of Italian nephrologists: an internet survey. J Nephrol 2016;29:385390. https://doi.org/10.1007/s40620-015-0232-y

11 Antonelli Incalzi R, Aucella F, Leosco D, et al. Assessing nephrological competence among geriatricians: a proof of concept internet survey. PLoS One 2015;10:e0141388. https://doi.org/10.1371/journal.pone.0141388

12 Ecarnot F, Pedone C, Cesari M, et al. Knowledge about vaccines and vaccination in older people: results of a national survey by the Italian Society for Gerontology \& Geriatrics. Vaccine 2020;38:1535-1540. https://doi.org/10.1016/j. vaccine.2019.11.065

13 Lane T, Fontana M, Martinez-Naharro A, et al. Natural history, quality of life, and outcome in cardiac transthyretin amyloidosis. Circulation 2019;140:16-26. https://doi. org/10.1161/CIRCULATIONAHA.118.038169

14 Costache II, Buburuz AM, Crisu D, et al. The role of echocardiography and 99mTc-HDP scintigraphy in noninvasive diagnosis of cardiac amyloidosis. Medicine (Baltimore) 2019;98:e17256. https://doi.org/10.1097/ MD.0000000000017256

15 Gillmore JD, Maurer MS, Falk $\mathrm{RH}$, et al. Nonbiopsy diagnosis of cardiac transthyretin amyloidosis. Circulation 2016;133:2404-2412. https://doi.org/10.1161/ CIRCULATIONAHA.116.021612

16 Ponikowski P, Voors AA, Anker SD, et al. 2016 ESC Guidelines for the diagnosis and treatment of acute and chronic heart failure. Eur J Heart Fail 2016;18:891-975. https://doi. org/10.1002/ejhf.592

17 Maurer MS, Schwartz JH, Gundapaneni B, et al. Tafamidis treatment for patients with transthyretin amyloid cardiomyopathy. N Engl J Med 2018;379:1007-1016. https:// doi.org/10.1056/NEJMoa1805689

18 Macedo AVS, Schwartzmann PV, de Gusmão BM, et al. Advances in the Treatment of Cardiac Amyloidosis. Curr Treat Options Oncol 2020;21. https://doi.org/10.1007/ s11864-020-00738-8

19 Kozhimannil KB, Karaca-Mandic P, Blauer-Peterson CJ, et al. Uptake and utilization of practice guidelines in hospitals in the United States: the case of routine episiotomy. The Joint Commission Journal on Quality and Patient Safety 2017;43:41-48. https://doi.org/10.1016/j. jcjq.2016.10.002 


\section{SUPPLEMENTAL FILES}

Supplemental Table I. Education and clinical attitudes for CA of the survey participants.

\begin{tabular}{|c|c|c|c|c|}
\hline & Residents (\%) & Specialists (\%) & All (\%) & $\mathbf{P}$ \\
\hline \multicolumn{5}{|c|}{ Where do you study clinical features, diagnosis and treatment of cardiac amyloidosis (more than one answer possible)? } \\
\hline PubMed & 78.8 & 76 & 78.1 & 0.642 \\
\hline Web pages and publications of the Ministry of Health & 16.3 & 17.3 & 16.5 & 0.927 \\
\hline Pharmaceutical company representatives & 5.2 & 21.2 & 9.4 & $<0.001$ \\
\hline Congresses and training courses & 21.8 & 31.7 & 24.4 & 0.059 \\
\hline International Scientific journals & 30.8 & 44.2 & 34.4 & 0.019 \\
\hline National scientific journals & 17.3 & 24 & 19.1 & 0.176 \\
\hline Individual web search & 58.8 & 41.3 & 54.2 & 0.003 \\
\hline Other & 0 & 0 & 0 & - \\
\hline \multicolumn{5}{|l|}{ Had you ever managed patients with cardiac amyloidosis? } \\
\hline No & 69.9 & 39.4 & 61.8 & $<0.001$ \\
\hline $\mathrm{Si}$ & 30.1 & 60.6 & 38.7 & \\
\hline \multicolumn{5}{|l|}{ If yes (more than one answer possible): } \\
\hline It was a first diagnosis & 11.4 & 17.3 & 13 & 0.173 \\
\hline It was a patient visited for other diseases & 14.5 & 35.6 & 20.1 & $<0.001$ \\
\hline It was a patient visited for symptoms of heart failure & 17.3 & 29.8 & 20.6 & 0.01 \\
\hline \multicolumn{5}{|l|}{ If you suspect cardiac amyloidosis: } \\
\hline $\begin{array}{l}\text { You always prescribe second level exams, independently } \\
\text { of age and comorbidities, with the exception of terminal } \\
\text { patients }\end{array}$ & 65 & 74.8 & 67.6 & 0.191 \\
\hline You prescribe second level exams only for younger patients & 1 & 1 & 1 & \\
\hline $\begin{array}{l}\text { You prescribe second level exams only for patients with } \\
\text { good performance status and few comorbidities }\end{array}$ & 33.9 & 24.3 & 31.4 & \\
\hline \multicolumn{5}{|c|}{ For the clinical management of cardiac amyloidosis (more than one answer possible): } \\
\hline Always ask for a cardiologic visit & 40.1 & 44.2 & 41.2 & 0.543 \\
\hline Always manage cardiologic symptoms independently & 8.1 & 19.2 & 11.1 & 0.004 \\
\hline $\begin{array}{l}\text { Manage cardiologic symptoms of older patients with } \\
\text { multimorbidity }\end{array}$ & 59.2 & 58.7 & 59 & 1 \\
\hline Ask for a cardiologic visit only for invasive procedures & 22.5 & 27.9 & 24 & 0.338 \\
\hline \multicolumn{5}{|c|}{ Do you usually prescribe screening exams for amyloidosis for all patients? } \\
\hline $\begin{array}{l}\text { Yes, if I have suggestive ECG and echocardiographic } \\
\text { features }\end{array}$ & 9.1 & 10.7 & 9.5 & 0.291 \\
\hline $\begin{array}{l}\text { Yes, if I have suggestive ECG and echocardiographic } \\
\text { features and/or electrophoresis }\end{array}$ & 45.6 & 45.6 & 45.6 & \\
\hline No, if heart failure treatment is not effective only & 24.9 & 31.1 & 26.5 & \\
\hline $\begin{array}{l}\text { Yes, if there are non-cardiac features suggestive for } \\
\text { amyloidosis }\end{array}$ & 20.4 & 12.6 & 18.3 & \\
\hline \multicolumn{5}{|l|}{ In your training, amyloidosis was studied: } \\
\hline During the MD & 59.7 & 26.2 & 50.9 & $<0.001$ \\
\hline During the fellowship & 6.9 & 21.4 & 10.7 & \\
\hline Both during the MD and the fellowship & 25 & 30.1 & 26.3 & \\
\hline Never, only occasional information during congresses & 8.3 & 22.3 & 12 & \\
\hline \multicolumn{5}{|l|}{ In your clinical practice, heart failure is: } \\
\hline Very common ( $>30 \%$ of cases) & 77.5 & 51 & 70.5 & $<0.001$ \\
\hline Common (10-30\% of cases) & 16.6 & 26.9 & 19.3 & \\
\hline Uncommon $(<10 \%$ of cases $)$ & 4.5 & 20.2 & 8.7 & \\
\hline Very uncommon & 1.4 & 1.9 & 1.5 & \\
\hline
\end{tabular}




\section{SUPPLEMENTAL TEXT 1. KNOWLEDGE SECTION OF THE SURVEY.}

1. Which is not a clinical sign expected in an 80-year-old patient with a recent diagnosis of cardiac amyloidosis?
a. Bilateral ankle swelling
b. Jugular veins distension
c. Bilateral carpal tunnel syndrome
d. High natriuretic peptides blood concentration

2. Which of the following ECG findings are characteristics of cardiac amyloidosis?
a. High voltages
b. Low voltages
c. Antero-septal $Q$ waves
d. Diffuse ST segment depression
e. $\mathrm{B}+\mathrm{C}$

3. Which of the following echocardiographic findings is characteristic of cardiac amyloidosis?
a. Bilateral ventricular hypertrophy
b. Atrial septum thickening
c. Reduced ejection fraction
d. Bilateral atrial enlargement
e. $A+B$

4. The prevalence of cardiac amyloidosis is:
a. About $20-50 / 100,000$
b. About $20-50 / 1,000.000$
c. $3 \%$ in people over 75 years of age
d. $0.5 \%$ in people over 80 years of age
e. $\mathrm{A}+\mathrm{C}$

5. Which of the following is not a common arrhythmic manifestation or a conduction system alteration of cardiac amyloidosis?
a. Sinus node disfunction
b. Atrio-ventricular block
c. Intra-ventricular conduction delay
d. Atrial fibrillation
e. QT prolongation

6. Which of the following sentences is FALSE:
a. AL and ATTR amyloidosis are the most frequent forms of cardiac amyloidosis
b. ATTRwt is more common in young people
c. Serum-protein electrophoresis has low sensitivity for AL form screening
d. Cardiac amyloidosis is often an underdiagnosed disease
e. Endomyocardial biopsy is the gold standard for the ATTR amyloidosis diagnosis

7. AL amyloidosis:
a. Is less aggressive than ATTR amyloidosis
b. Can affect other organs than heart
c. From heart failure symptoms onset, if not treated, has a mean survival $<6$ months
d. $\mathrm{A}+\mathrm{B}$
e. $B+C$ 
8. Which of the following sentences on ATTR amyloidosis is FALSE:
a. It has 2 variants, the ATTRwt and the ATTRm
b. The ATTRwt is an autopsy finding in $25 \%$ of over 85 years old people
c. The ATTRwt is more common among women
d. The ATTRm included many different mutations
e. It should be often suspected in older adults with hypertrophic cardiopathy

9. Which of the following sentences is FALSE? In ATTRwt amyloidosis patients:
a. Atrial fibrillation onset cannot be years before diagnosis
b. Spinal canal stenosis can occur 5-15 years before cardiac symptoms
c. Heart failure symptoms are often the first systemic manifestation of the disease
d. Heart failure is only diastolic
e. $A+B$

10.If you suspect cardiac amyloidosis, the serum and urinary immunofixation:
a. Is not useful if serum-protein electrophoresis is negative
b. Is indicated if you suspect $A L$ amyloidosis
c. In the AL form shows an alteration of the kappa/lambda ratio
d. In the AL form shows a preserved kappa/lambda ratio
e. $B+C$

11.For the instrumental diagnosis of amyloidosis:
a. The cardiac magnetic resonance is not useful if performed without contrast agent
b. The cardiac magnetic resonance, despite very promising, has low sensitivity and specificity
c. The strain imaging shows diffuse alterations of left ventricular walls
d. The strain imaging shows an apical sparing pattern
e. The ECG shows typical alteration in $80 \%$ of cases

12.A 90-year-old patient, affected from many years by hypertension, type II diabetes, and atrial fibrillation, comes for a visit because of worsening ankle swelling and dyspnea. He has also a previous history, many years before, of carpal tunnel syndrome. Do you think that:
a. Considering his age, a new diagnosis of cardiac amyloidosis is unlikely
b. It is necessary a clinical evaluation including ECG and echocardiography
c. You can diagnose cardiac amyloidosis with echocardiography only
d. If scintigraphy documents a grade 3 uptake, the diagnosis of cardiac amyloidosis is unlikely
e. Scintigraphy is not useful if you suspect the ATTRwt form

13. You can diagnose ATTRwt amyloidosis with:
a. Clinical evaluation + ECG + echocardiography
b. Endomyocardial biopsy
c. Right cardiac catheterization
d. Periumbilical fat biopsy
e. $A+B+D$

14. Diuretic treatment in patients affected by cardiac amyloidosis:
a. Should include also thiazide diuretics
b. Should prefer torasemide to furosemide
c. Should not include aldosterone blockers because of a high risk of kidney failure
d. Has etacrynic acid as the first choice
e. $\mathrm{B}+\mathrm{C}$ 
15.Which of these drugs ARE NOT recommended in patients with cardiac amyloidosis?
a. B-blockers
b. Calcium-channel blockers
c. Digoxin
d. Nitrates
e. $\mathrm{B}+\mathrm{C}$

16. Which of the following sentences is FALSE? The target of the treatment of ATTR amyloidosis is:
a. To block hepatic protein synthesis of TTR
b. To stabilize TTR tetramers
c. To stabilize TTR dimers
d. To remove amyloid fibrils
e. None of the precedents

17. Clinical classifications of ATTR amyloidosis are based on:
a. Troponin and NT-proBNP values
b. Creatinine and NT-proBNP values
c. Troponin and creatinine values
d. $\mathrm{CK}-\mathrm{MB}$ and creatinine values
e. $A+B$

18 Is it true that the association between aortic valve stenosis and cardiac amyloidosis interests $30 \%$ of patients with aortic valve stenosis?
a. Yes, in low-flow low-gradient aortic valve stenoses
b. Yes
c. No
d. Among men
e. If aortic valve stenosis is associated with atrial fibrillation

19.A high myocardial uptake at bone scintigraphy is suggestive of:
a. AL cardiac amyloidosis
b. TTR cardiac amyloidosis
c. TTRwt cardiac amyloidosis
d. Hereditary TTR cardiac amyloidosis
e. Cardiac amyloidosis

20.Which of the following sentences on Tafamidis is TRUE:
a. Stabilizes TTR tetramers slowing their dissociation in monomers
b. Blocks hepatic synthesis of TTR
c. Is not effective for the treatment of ATTRh polyneuropathy
d. Has the same mechanism of action of Doxiciclin
e. The is not available evidence on its efficacy in improving survival of patients affected by cardiac amyloidosis 